\section{DIVISION G COMMISSION 29}

\section{STELLAR SPECTRA \\ SPECTRES STELLAIRES}

\author{
Katia Cunha \\ David R. Soderblom \\ Nikolai Piskunov \\ Wako Aoki, Martin Asplund, \\ Kenneth G. Carpenter, \\ Paul Crowther, \\ Jorge Melendez, Kimberly Venn, \\ Vanessa Hill, David Yong
}

\title{
Highlights of IAU Commission 29: Recent Advances and Perspectives on Stellar Spectroscopy
}

\section{Introduction}

IAU Commission 29 - Stellar Spectra has been one of the IAU commissions from the onset, until its dissolution at the most recent IAU General Assembly in Honolulu in 2015. This commission belonged to IAU Division G ("Stars and Stellar Physics"), the latter committed with fostering research in stellar astrophysics. Within the general field of stellar astrophysics, stellar spectroscopy plays a key role, as stellar spectra are a powerful tool providing a view into the detailed physical properties of stars and the physical processes occuring within them.

The scope of IAU Commission 29 has been quite broad, from instrumentation in spectroscopy, to stellar spectral analysis methods, as well as stellar atmospheres modeling to provide not only the crucial stellar parameters, such as effective temperatures and surface gravities, but also to decode the chemical information from the stellar photospheres. Because of its history, Commission 29 has predominantly been related to optical spectroscopy, but has expanded to include the infrared and ultraviolet as detector technology and observations from space extended spectroscopic capabilities into these domains.

With such an over-arching scope, Commission 29 has been one of the largest IAU commissions having had $\sim 500$ members. In addition, IAU Commission 29 had synergy with many other IAU working groups and commissions dealing with different aspects of stellar astrophysics, such as:

Commission 26 - Double and Multiple Stars;

Commission 35 - Stellar Constitution;

Commission 36 - Theory of Stellar Atmospheres;

Commission 42 - Close Binary Stars;

Commission 45 - Stellar Classification. 
This is the last report on the activities of IAU Commission 29, which at the time of this writing no longer exists, given the latest re-structuring of the IAU commissions and working groups. In this contribution to the legacy volume of the IAU, we will first highlight a few historical aspects of IAU Commission 29 and then briefly discuss significant achievements and a few selected results in the field of stellar spectroscopy within the last few years. It should be noted, however, that this is a mostly personnal and certainly biased view of the ex-president (Cunha) and vice-president (Soderblom) of IAU Commission 29, with contributions from present and past organizing committee members, as indicated in the text.

\section{A Few Historical Aspects}

IAU Commission 29 was one of the original 32 commissions that were established by the IAU in 1919. Its original name was "Spectral Classification" and its first President was Walter S. Adams. As the classification of stellar spectra became increasingly important and the analysis of stellar spectra from modeling also became increasingly more sophisticated, a new IAU commission was founded during the IAU GA in Hamburg in 1964: IAU Commission 45. This new commission 45 would then focus on, and take the name "Spectral Classification", while Commission 29 would then become "Stellar Spectra" (Trimble, 2015).

A list of former IAU Commission 29 Presidents (P) and Vice-Presidents (VP) for triennial terms since the early 1970's has been compiled by Virginia Trimble and is presented below. In the IAU organization, the Vice-President of a commission is elected by Commission members and she/he becomes president of the Commission in the following term when a new Vice-President is elected.

1973-1976 C29 P: Alexander A. Boyarchuk, VP: Margherita Hack

1976-1979 C29 P: Margherita Hack, VP: Walter K. Bonsack

1979-1982 C29 P: Walter K. Bonsack, VP: Jun Jugaku

1982-1985 C29 P: Jun Jugaku, VP: Giusa Cayrel de Strobel

1985-1988 C29 P: Giusa Cayrel de Strobel, VP: Peter S. Conti

1988-1991 C29 P: Peter S. Conti, VP: David L. Lambert

1991-1994 C29 P: David L. Lambert, VP: Michael S. Bessel

1994-1997 C29 P: Michael S. Bessell, VP: Beatriz Barbuy

1997-2000 C29 P: Beatriz Barbuy, VP: Gautier Mathys

2000-2003 C29 P: Gautier Mathys, VP: Christopher Sneden

2003-2006 C29 P: Christopher Sneden, VP: Mudumba Parthasarathy

2006-2009 C29 P: Mudumba Parathasarathy, VP: Nikolai Piskonov

2009-2012 C29 P: Nikolai Piskunov, VP: Katia Cunha

2012-2015 C29 P: Katia Cunha, VP: David R. Soderblom

As can be seen from the list above, within the past four decades, the participation of women in leadership roles in C29 represents $\sim 28 \%$ : 4 women became president in 14 triennial terms. The geographical representation of the Commission Presidents has been as follows: Australia (1); South America: Brazil (2), Chile (1); North America: USA (4); Europe: Italy (1), France (1), Sweden (1); USSR (1); Asia: India (1); Japan (1). 


\section{Science Highlights}

Summaries of IAU Commission 29 activities within the past decade can be found in previous triennial reports by Piskunov et al. (2012), Parthasarathy et al. (2009), and Sneden et al. (2007). These reports include science highlights, as well as lists of IAU meetings sponsored by the commission (these are mainly sponsored at the Division level in the current structure of the IAU). A few science highlights for Commission 29 over the last few years are discussed below.

\subsection{Stellar Spectroscopy in the Era of Large Surveys: Cartography of the Milky Way}

The last years have been witness to a vast increase in the amount of spectroscopic data available for undertaking chemo-dynamical studies in the Milky Way. Until recently, most spectroscopic studies of Galactic stars had been traditionally limited to observations obtained one-at-a-time of carefully selected objects. This modus operandi is very expensive and results in relatively small samples of stars with good quality spectra. A significant change in this paradigm has happened. A dramatic increase in data flow has stemmed from major developments in instrumentation resulting in innovative designs of multiplexed and highly efficient multi-object spectrographs deployed on dedicated mid-size telescopes observing in survey mode, and in the availability of resources needed to undertake such large-scale spectroscopic surveys by associating large consortia of astronomers around the globe.

Several spectroscopic surveys are currently probing the kinematic and chemical structure of the Milky Way in unprecedented detail. There are low, medium and high spectral resolution surveys in the optical and infrared that have already observed close to one million Galactic stars of all major components of the Milky Way, which include the bulge, thick and thin disks, and halo. Completed spectroscopic surveys include the optical medium resolution RAVE survey (RAdial Velocity Experiment; Steinmetz et al. 2006), the optical low-resolution surveys SEGUE-1, -2 (Sloan Extension for Galactic Understanding and Exploration; Yanny et al. 2009; Rockosi et al. 2015), and the near-infrared high-resolution survey APOGEE-1 (Apache Point Observatory Galactic Evolution Experiment; Majewski et al. 2015); SEGUE-2 and APOGEE were both part of the third Sloan Digital Sky Survey (SDSS-III).

On-going optical surveys include the LAMOST survey (Large Sky Area Multi-Object Fibre Spectroscopic Telescope; Zhao et al. 2012), the GALAH survey (GALactic Archaeology with Hermes; De Silva et al. 2015) on the Anglo-Australian telescope, and the Gaia/ESO survey (GES; Gilmore et al. 2012, Randich et al. 2013) on the ESO VLT. In addition, there is the SDSS-IV APOGEE-2 survey (Majewski et al. 2015) in the nearinfrared, an extension of the APOGEE-1 survey, continuing observations at the Apache Point Observatory and adding a southern hemisphere component with a second APOGEE spectrograph on the Du Pont telescope. The combination of optical and infrared spectroscopy from the northern and southern hemispheres is fundamental in reaching all parts of the Galaxy, including regions which are heavily obscured by dust. This ability to probe large volumes of the Galaxy is currently achieved with a combination of both access to a larger aperture telescope (Gaia-ESO) and observations in the near-infrared (APOGEE). In addition, both Gaia-ESO and APOGEE have high spectral resolution, providing the opportunity to probe the chemistry of several elements in great detail. This matrix is creating an ever more complete map of the Galaxy in terms of kinematics and detailed chemical content, via the determination of radial velocities and chemical abundances of a large number of elements having distinct nucleosynthetic origins. The chemical 
information within all of these stars is being used to identify the dispersed stellar building blocks that went into forming the major stellar populations of the Milky Way.

Future multiplexed spectroscopic facilities and accompanying surveys are being planned and built and these will continue to provide large amounts of spectroscopic data of Galactic stars. Such surveys include the Galactic components of 4MOST (4metre Multi-Object Spectroscopic telescope; de Jong et al. 2014) on the VISTA telescope, WEAVE (wide-field multi-object spectrograph; Balcells et al. 2010) on the William Herschel telescope, DESI (Dark Energy Spectroscopic Instrument; Poppett et al. 2015) on the Kitt Peak 4m telescope, PFS (Prime Focus Spectrograph; Takada et al. 2014) on the Subaru telescope, as well as the optical and near-infrared survey MOONS on the VLT (Cirasuolo et al. 2014).

\subsection{Synergy Between Large Spectroscopic Surveys and Asteroseismology (Verne Smith)}

Although spectroscopy is a powerful tool for deriving stellar physical properties and for extracting chemical abundances, the key properties of mass and age are only indirectly (and weakly) constrained by spectroscopy. Asteroseismology, on the other hand, can provide, through global oscillations, fairly accurate values of stellar mean density and surface gravity (Stello et al. 2009; Huber et al. 2011). If these are combined with stellar effective temperature $\left(\mathrm{T}_{\text {eff }}\right)$ and chemical composition (metallicity), the stellar mass and radius can be derived, with values that are more accurate than have generally been available to date.

With the CoRoT (De Ridder et al. 2009) and Kepler (Bedding et al. 2010) space missions, red giant oscillations have been measured for large samples of these types of stars. For targets in common with spectroscopic surveys, the oscillation data can be combined with the spectroscopic values of $\mathrm{T}_{\text {eff }}$ and $[\mathrm{Fe} / \mathrm{H}]$ (along with $[\alpha / \mathrm{Fe}]$ ), to derive masses (and hence ages) for samples of red giants. The synergy between red giant oscillations and large survey spectroscopic results has been exploited by Kepler and APOGEE for 1916 red giants in combined Kepler and APOGEE fields (Pinsonneault et al. 2014). The combination of APOGEE and Kepler data results in quite accurate values for $\mathrm{T}_{\mathrm{eff}}$, log g, red giant mass (hence age), and type (i.e., first-ascent giant or clump giant), along with detailed chemical compositions of $\sim 15$ elements. CoRoT and APOGEE results have also been combined (e.g., Chiappini et al. 2014) to investigate chemical enrichment, age and radial migration.

\subsection{Globular Clusters and Metal-Poor Stars (David Yong)}

Globular clusters have long been regarded as the best examples of simple stellar populations, i.e., a single age, helium abundance, metallicity, and initial mass function. Beginning with $\omega$ Centauri, however, there is a small, but growing class of globular clusters that exhibit dispersions in metallicity. In many cases, there are large star-to-star abundance variations for the slow neutron-capture process elements. Within the past three years, the following clusters have been identified as harboring metallicity and $s$-process element variations: M2 (Yong et al. 2014), NGC 5286 (Marino et al. 2015) and NGC 6273 (Johnson et al. 2015). These stellar systems are fascinating objects in which to study $s$ process nucleosynthesis at low metallicity and may have cosmological implications as the remnants of dwarf galaxies.

There has also been significant effort in searching for metal-poor stars in the bulge. Cosmological simulations predict that the the first stars to have formed are expected to now reside in the central regions of the Galaxy rather than in the halo (e.g., Tumlinson 
2010). Recent searches for metal-poor stars in the bulge have been successful in finding stars with $[\mathrm{Fe} / \mathrm{H}]<-2.5$ (Howes et al. 2014; Casey \& Schlaufman 2015 and Howes et al. 2015). While not necessarily the most metal-poor stars known, these objects are potentially the oldest stars in our Galaxy. It is worth highlighting, also, that the most Fe-poor star found to date (Keller et al. 2014) with a metallicity (iron abundance) of $[\mathrm{Fe} / \mathrm{H}]<-7.1$, has an enhanced carbon abundance, as is the case for the vast majority of the extremely low metallicity stars in the Galaxy, which have very high values of $[\mathrm{C} / \mathrm{Fe}]$, $[\mathrm{N} / \mathrm{Fe}]$, and $[\mathrm{O} / \mathrm{Fe}]$.

\subsection{Extrasolar Planets and the Chemistry of Stars with Planets}

High-resolution spectroscopic analyses of the host stars of exoplanet systems continues to be an active area of stellar astrophysics. It has been known from the early radial-velocity discoveries that stars which host giant planets tend to have rather large metallicities (Gonzalez 1997; Santos et al. 2004). Fischer \& Valenti (2005) found that the fraction of FGK dwarfs hosting closely orbiting giant planets (where the stellar reflex vsini > $30 \mathrm{~m} / \mathrm{s}$ ) increases dramatically with $[\mathrm{Fe} / \mathrm{H}]$. The giant planet metallicity connection has been confirmed with more recent studies and independent analyses (e.g., Ghezzi et al. 2010, Sousa et al. 2011).

Recently, the relationship between exoplanet size and host-star metallicity has been extended beyond giant planets to include a broader range of exoplanet sizes. For example, Buchhave et al. (2014) have mapped the planet-size/metallicity relation in more detail by analyzing 600 exoplanets around 400 host stars identified by Kepler. They found 3 classes of planet sizes that can be identified with distinct metallicity regimes. Terrestriallike planets $\left(\mathrm{R}<1.7 \mathrm{R}_{\text {Earth }}\right)$ tend to be found around stars with the lowest average metallicity $(<[\mathrm{M} / \mathrm{H}]>=-0.02 \pm 0.02)$, while 'gas-dwarf' planets $\left(\mathrm{R}=1.7-3.9 \mathrm{R}_{\text {Earth }}\right)$ have host stars with $<[\mathrm{M} / \mathrm{H}]>=+0.05 \pm 0.01$, and 'gas-giant' planets $\left(\mathrm{R}>3.9 \mathrm{R}_{E \text { arth }}\right)$ are found with stars having $\langle[\mathrm{M} / \mathrm{H}]\rangle=+0.18 \pm 0.02$. Buchhave et al. (2014) conclude that host-star metallicity sets the amounts of available solids in a protoplanetary disk, which is a key variable in setting the mass distributions of planets. However, Wang \& Fischer (2015) find a different relation than Buchhave et al. (2014), deriving an increasing likelihood of planets of all sizes with increasing metallicity, although still finding that smaller planets have a weaker trend with metallicity than giant planets. Schuler et al. (2015) conducted precision abundance analyses of 7 stars hosting small rocky planets discovered by Kepler and their results indicate that such planets can form around stars having a range of chemical abundance distributions and metallicities.

Pursuing exoplanet host-star chemistry in detail will continue to be important as the abundances of certain key solid-forming elements, such as Si and Mg (Brugamyer et al. 2011; Adibekyan et al. 2015), or the ratio of C/O, which controls ice chemistry (e.g., Teske et al. 2014), are suggested to affect the nature of planetary system architecture. Adibekyan et al. $(2012 ; 2014)$ have found that certain elements, such as Ti, increase the likelihood of giant planet occurrence and suggest that the Galactic thick-disk population, which is slightly Fe-poor but $[\alpha / \mathrm{Fe}]$ enhanced, hosts a larger fraction of exoplanet systems than the thin disk. The recent precise abundance analysis of Nissen (2015), for example, finds correlations of specific abundance ratios between volatile and refractory elements that depend on stellar age. It may be that condensation temperature abundance effects once thought to be due to the presence of exoplanets may depend more on the age and Galactic birthplace of the host star. 


\subsection{Planet Formation and Galactic Chemical Evolution (Jorge Melendez)}

It seems well established that metallicity is not the only parameter that influences the abundance pattern of stars in the Galactic disks. The best example of this is our Sun, that shows a distinct chemical abundance pattern relative to solar twins, which are stars with stellar parameters similar to the Sun (e.g., Melendez et al. 2009). Initially the solar abundance pattern was ascribed to a planet signature, but the recent high precision $(0.01$ dex) work on solar twins by Nissen (2015) shows that even for stars of the same (solar) metallicity, there are correlations with both age (chemical evolution effects) and condensation temperature (planet effects). The deficiency of refractory elements in the Sun could be thus explained by both Galactic chemical evolution and planet formation/evolution. Furthermore, the precise work of Nissen (2015) shows that the [Y/Mg] ratio could be used as a proxy for stellar age.

\subsection{Spectroscopic Follow-up Efforts}

The drive to find, characterize, and understand exoplanets has placed severe demands on the need for spectroscopic follow-up observations, and has led to higher levels of precision and accuracy than ever before. In addition, the need for stellar characterization of planet hosting stars adds to the demand for spectroscopy in the exoplanet field: "One only knows the planet as well as one knows the hosting star" and determining the stellar radius is necessary in order to infer the planet's radius. Exoplanets discovered via transit (the majority of the exoplanet discoveries to date), for example, by the Kepler mission, both Kepler prime and $\mathrm{K} 2$, as well as, the CoRoT mission would still need to be confirmed, mostly via spectroscopy. Results from the Kepler mission have shown that most stars have extrasolar planets (Batalha 2014). Radial velocities continue to be essential for characterizing exoplanets. Transits only reveal a planet's size, while radial velocity measurements are necessary to measure its mass, and thus the planets density and composition.

\subsection{The Future}

Radial velocity measurements at the level needed to detect Earth-mass exoplanets require control of spectrograph properties at or near the limit of capability. Innovative designs and extensive engineering are enabling the meter-per-second level accuracy needed in order to detect earth-mass planets. At such level the intrinsic stellar signal most likely interferes and this is a challenge for the desire to find such small planets via radial velocity measurements. It is clear, however, that improvements in spectrograph performance and spectral analysis will both benefit stellar and exoplanet studies. For example, both very high-resolution and spectrograph stability are needed to measure the asymmetries of absorption lines in late-type stars that arise from convection. Convection controls the dynamo mechanism in the Sun and solar type stars, yet remains poorly understood.

Concerning the understanding of our home Galaxy, the on-going Gaia mission (Perryman et al. 2001) will certainly change the landscape in the near future with knowledge of the key parameter of distance, which for field stars currently has very large uncertainties, at best. With distances in hand, the ages of stars will also be known to better precision, and these results, allied with results from the ongoing and future large spectroscopic surveys (Section 3.1), will create a map of the Galaxy in unprecedented detail.

The three planned Extreme Large Telescopes (ELT's), the Giant Magellan Telescope (GMT), the European Extremely Large Telescope (E-ELT), and the Thirty Meter Telescope (TMT) have spectroscopic capabilities planned for their instrumentation. 
Currently, the ELT's are not planned to be dedicated survey telescopes nor to conduct large surveys, but they will have the capability to target particularly interesting objects, families of objects, or interesting questions discovered by the large surveys. Therefore, the ELT spectroscopic capabilities will complement the survey science on the smaller telescopes and it is through quantitative spectroscopy that the greatest progress will be made over the coming decades.

Several future missions will continue to probe stars in the Milky Way throughout all locations in the Galaxy, and, as we now know, most stars likely host planets. The modeling of the atmospheres of stars and of the atmospheres of surrounding planets will shed light on several aspects of planet formation. Spectroscopy is the link that connects all this detailed knowledge of the physical processes involved. These observational developments, however, would be of limited use if not accompanied by advances in the theory and modeling of stellar and planetary atmospheres such that precise and accurate physical information can be extracted from the observed spectra. Concerted efforts from both observations and theory will significantly advance our knowledge of stellar astrophysics, planetary formation and galaxy formation and evolution.

\section{References}

Adibekyan, V. Zh., Sousa, S. G., Santos, N. C., et al. 2012, A\&A, 545, 32

Adibekyan, V., Santos, N. C., Figueira, P., et al. 2015, A\& A, 581, L2

Balcells, M., Benn, C. R., Carter, D., et al. 2010, vol. 7735 of Society of Photo-Optical Instrumentation Engineers (SPIE) Conference Series, 7

Batalha, N. M. 2014, PNAS, 11112647

Bedding, T. R., Huber, D., Stello, D., et al. 2010, ApJL, 713, L176

Brugamyer, E., Dodson-Robinson, S. E., Cochran, W. D., \& Sneden, C. 2011, ApJ, 738, 97

Buchhave, L. A., Bizzarro, M., Latham, D. W., et al. 2014, Nature, 509, 593

Casey, A. R. \& Schlaufman, K. C. 2015, ApJ, 809, 110

Chiappini, C., Anders, F., \& Minchev, I. 2014, EAS Publications Series, Volume 67-68, pp.169176

Cirasuolo, M., Afonso, J., Carollo, M., et al. 2014, SPIE, 9147

De Ridder, J., Barban, C., Baudin, F., et al. 2009, Nature, 459, 398

De Silva, G. M., Freeman, K. C., Bland-Hawthorn, J., et al. 2015, MNRAS, 449, 2604

de Jong, R. S., Barden, S., Bellido-Tirado, O., et al. 2014, vol. 9147 of Society of Photo-Optical Instrumentation Engineers (SPIE) Conference Series, 0

Fischer, D. A. \& Valenti, J. 2005, ApJ, 622, 1102

Ghezzi, L., Cunha, K., Smith, V. V., de Araujo, F. X., Schuler, S. C., \& de la Reza 2010, ApJ, 720,1290

Gilmore, G., Randich, S., Asplund, M., et al. 2012, The Messenger, 147, 25

Gonzalez, G. 1997, MNRAS, 285, 403

Howes, L. M., Asplund, M., Casey, A. R., et al. 2014, MNRAS, 445, 4241

Howes, L. M., Casey, A. R., Asplund, M., et al. 2015, arXiv151103930H

Huber, D., Bedding, T. R., Stello, D., et al. 2011, ApJ, 743, 143

Johnson, C. I., Rich, R. M., Pilachowski, C. A., et al. 2015, AJ, 150, 63

Keller, S. C., Bessell, M. S., Frebel, A., et al. 2014, Nature, 506, 463

Majewski, S. R., Schiavon, R. P., Frinchaboy, P. M., et al. 2015, arXiv150905420M

Marino, A. F., Milone, A. P., Karakas, A. I., et al. 2015, MNRAS, 450, 815

Melendez, J., Asplund, M., Gustafsson, B., \& Yong, D. 2009, ApJ, 704, 66

Nissen, P. E. 2015, A\&\&A, 579, 52

Parthasarathy, M., Piskunov, N. E.; Sneden, C., et al. 2009, Transactions IAU, Volume 4, Issue 27A, Reports on Astronomy 2006-2009. Edited by Karel van der Hucht. Cambridge: Cambridge University Press, 2009, p. 209-210

Perryman, M. A. C., de Boer, K. S., Gilmore, G., 2001, A\& $A$, 369, 339 
Pinsonneault, M. H., Elsworth, Y., Epstein, C., et al. 2014, ApJS, 215, 19

Piskunov, N. E., Cunha, K., Parthasarathy, M. et al. 2012, Transactions IAU, Volume 7, Issue T28A, p. $157-160$

Poppett, C., \& DESI Collaboration 2015, vol. 225 of AAS Meeting Abstracts, 413.04

Randich, S., Gilmore, G., \& Gaia-ESO Consortium 2013, The Messenger, vol. 154, p. 47-49

Rockosi, C. et al. 2015, in preparation

Santos, N. C., Israelian, G., \& Mayor, M. 2004, A\&A, 415, 1153

Sneden, C., Parthasarathy, M., Castelli, F., et al. 2007, IAU Transactions, Vol. 26A, Reports on Astronomy 2002-2005. Edited by O. Engvold. Cambridge: Cambridge University Press, 2007., pp.203-204

Sousa, S. G., Santos, N. C., Israelian, G., Mayor, M., \& Udry, S. 2011, A\&A A, 533, 141

Steinmetz, M., Zwitter, T., Siebert, A., et al. 2006, AJ, 132, 1645

Stello, D., Chaplin, W. J., Basu, S., Elsworth, Y., \& Bedding, T. R. 2009, MNRAS, 400, L80

Takada, M., Ellis, R. S., Chiba, M., et al. 2014, PASJ, 66, 1

Teske, J. K., Cunha, K., Smith, V. V., Schuler, S. C. \& Griffith, C. A. 2014, ApJ, 788, 39

Trimble, V. 2015, private communication

Tumlinson, J. 2010, ApJ, 708, 139

Wang, J. \& Fischer, D. A. 2015, AJ, 149, 14

Yanny, B., Rockosi, C., Newberg, H. J., et al. 2009, AJ, 137, 4377

Yong, D., Roederer, I. U., Grundahl, F., et al. 2014, MNRAS, 441, 3396

Zhao, G., Zhao, Y.-H., Chu, Y.-Q., Jing, Y.-P., \& Deng, L.-C. 2012, Research in Astronomy and Astrophysics, 12, 723 